\title{
Perfil epidemiológico de idosos frequentadores de grupos de convivência no município de Iguatu, Ceará
}

Epidemiologic profile of elderly members of community groups in the city of Iguatu, Ceará

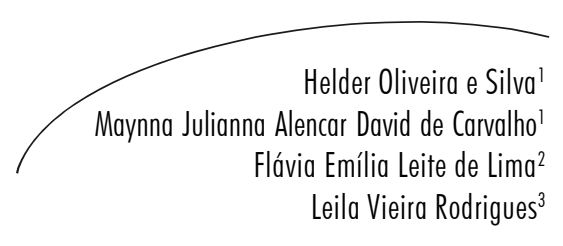

O envelhecimento da população brasileira nas últimas décadas propiciou a criação e efetivação de políticas públicas voltadas para a garantia dos direitos sociais da pessoa idosa. Tornaram-se crescentes as necessidades de implantar estratégias que propiciem autonomia, integração social e qualidade de vida para este grupo etário. O presente estudo objetivou descrever o perfil epidemiológico dos idosos que participam de grupos sociais da terceira idade do Município de Iguatu - CE. Trata-se de um estudo transversal, com abordagem quantitativa. A amostra foi composta por 65 idosos participantes de dois grupos de convivência do município. A coleta de dados foi realizada no período de fevereiro e março de 2009. Os resultados apontaram que a idade média dos entrevistados foi de 69,8 anos $( \pm 6,62), 59(90,8 \%)$ eram mulheres, 29 (44,6\%) eram viúvos e $26(40 \%)$ idosos possuíam entre 4 e 7 anos de estudo. $47,7 \%(\mathrm{n}=31)$ sustentavam sozinhos suas residências; $70,7 \%(n=46)$ revelaram não ter vida sexual ativa; $84,6 \%(n=55)$ participavam de outras atividades de lazer extra-grupo; $7,7 \%(\mathrm{n}=05)$ eram fumantes e 9,2\% ( $\mathrm{n}=06)$ faziam uso de bebida alcoólica. 90,8\% $(\mathrm{n}=59)$ realizavam um ou mais tipos de exercício físico e 53,8\% $(\mathrm{n}=35)$ avaliaram sua saúde como regular. Conclui-se que a participação em grupos de convivência permite que a pessoa idosa se mantenha ativa e incluída nas atividades sociais, promovendo troca de experiências e melhorando sua autoestima e qualidade de vida.

\section{Abstract}

The aging population in recent decades allowed the creation and fulfillment of public policies to guarantee the social rights of the elderly. There an increasing need dor strategies that provide autonomy, social integration and quality of life for this age group. This study aimed to describe the epidemiological profile of older people participating in social groups of the third age in the City of Iguatu

\footnotetext{
1 Universidade Regional do Cariri. Centro de Ciências Biológicas e da Saúde Curso de Enfermagem. Crato, CE, Brasil.

2 Universidade Federal de Sergipe, Núcleo de Nutrição. Aracajú, SE, Brasil.

3 Programa Saúde da Família. Prefeitura Municipal de Fortaleza. Fortaleza, CE., Brasil
}

Correspondência / Correspondence

Helder Oliveira e Silva

E-mail: heldersilva@terra.com.br.
Palavras-Chave:

Envelhecimento. Grupo de Idosos. Qualidade de Vida. 
- CE. This is a cross-seccional study, with quantitative approach. The sample consisted of 65 elderly participants in two groups living in the municipality. Data was collected in February-March 2009. The results show that $90.8 \%(n=59)$ of respondents were women, mean age was 69.8 years $(\mathrm{SD} \pm 6.62), 44.6 \%(\mathrm{n}=29)$ were widowed and $40 \%(\mathrm{n}=26)$ of the elderly had between 4 and 7 years of schooling; $76.9 \%(\mathrm{n}=50)$ of the sample reported having enough money to supply their basic needs and $47.7 \%(\mathrm{n}=31)$ maintained their own homes; $70.7 \%(\mathrm{n}=46)$ reported no active sexual life; $84.6 \%(\mathrm{n}=55)$ participated in other leisure activities outside the group, $7.7 \%(\mathrm{n}=05)$ were smokers and $9.2 \%(\mathrm{n}=06)$ were using alcohol. $90.8 \%(\mathrm{n}=59)$ performed one or more types of exercise and $53.8 \%(\mathrm{n}=$ 35) evaluated their health as regular. It was concluded that participation in groups together allows the elderly to remain active and included in social activities, promoting exchange of experiences and improving their self-esteem and quality of life.
Key words: Aging. Seniors Group. Quality of Life.

\section{INTRODUÇÃO}

O envelhecimento da população idosa brasileira é um fenômeno recente e está fortemente atrelado à melhoria da qualidade de vida, à tendência de diminuição do crescimento populacional, ao melhor controle dos agravos e uma intensa urbanização desse grupo etário. Esse crescimento da população idosa demanda ações em diversos setores da sociedade, na busca de atender de forma digna às necessidades inerentes dessa faixa etária mais avançada.

A Organização Mundial da Saúde (OMS) define a população idosa como aquela a partir dos 60 anos de idade. Esse limite é válido para os países em desenvolvimento, mas admite-se um ponto de corte de 65 anos de idade para os países desenvolvidos, pela tradição destes em utilizarem esse índice há várias décadas. ${ }^{1}$

A caracterização das condições de saúde dos idosos requer informações detalhadas sobre diferentes aspectos da vida desses indivíduos. Nos países desenvolvidos, é crescente o número de investigações que abordam as associações entre a saúde dos idosos e os determinantes demográficos e socioeconômicos, as doenças crônicas e a capacidade funcional. Uma forma de conduzir esse tipo de estudo é o levantamento de informações sobre a qualidade de vida e as condições do estado de saúde, considerando diversos aspectos da vida do indivíduo.
Um envelhecimento saudável depende da interação multidimensional de vários fatores. No entanto, são poucos os trabalhos que exploram um modelo que combine a idade, o sexo, o arranjo familiar, o estado conjugal, a educação, a renda, as doenças crônicas e a capacidade funcional, especialmente nos países em desenvolvimento, como é o caso específico do Brasil. ${ }^{2}$

Dessa forma, torna-se relevante realizar estudos que investiguem e discutam as condições de vida, de saúde e de suporte social dos idosos, para que se possa estar preparado para atender às demandas sociais, sanitárias, econômicas e afetivas dessa parcela da população que, atualmente, é a que mais cresce em termos proporcionais.

Com a finalidade de obter subsídios que possibilitem o planejamento local das ações, o presente estudo objetiva descrever o perfil epidemiológico dos idosos que participam grupos sociais da terceira idade do município de Iguatu $\mathrm{CE}$, no intuito de conhecer as condições socioeconômicas, a dinâmica familiar e a autoavaliação de saúde dessa população.

\section{MÉTODOS}

Trata-se de um estudo transversal com abordagem quantitativa. A pesquisa foi realizada no município de Iguatu, localizado na região Centro-Sul do Estado do Ceará, a 380 km da 
capital Fortaleza e com uma população de 92.260 habitantes. ${ }^{3}$ Participaram do estudo idosos a partir de 60 anos que frequentavam dois grupos de convivência de idosos na cidade, promovidos por instituições da sociedade civil. O primeiro grupo, conhecido como Grupo de Idosos do SESC, foi fundado no ano de 1996 e possui uma média de 64 idosos participantes. Os encontros são realizados todas as quintas-feiras à noite. As principais atividades realizadas são oficinas sobre a educação alimentar direcionada para a qualidade de vida, dança, artesanato, teatro e canto em coral. O grupo encontra-se também duas vezes na semana para realização de exercícios físicos orientados por um profissional de educação física. Em datas comemorativas são realizados passeios que contam com a participação dos familiares dos idosos, que são também convidados mensalmente para participar de ações integrativas com o grupo.

O segundo grupo, denominado Melhor Idade, foi fundado no ano de 2006, contando atualmente com 26 idosos participantes. Os encontros são realizados nas segundas, quartas e sextas-feiras no início da manhã. As atividades englobam alongamentos, caminhada, treinamento localizado, dinâmicas de grupo, prática de capoeira adequada para a idade, relaxamento e ginástica aeróbia. Estas são supervisionadas e orientadas por dois profissionais de educação física. Possuem também atividades extras, que são trabalhos artesanais, palestras, passeios, danças folclóricas e teatro. O grupo conta ainda com um profissional de enfermagem que faz a aferição da pressão arterial sempre que possível.

O presente estudo foi composto por 65 idosos frequentadores de dois grupos de convivência existentes no município. Os idosos foram pessoalmente convidados e selecionados por conveniência. Os critérios de inclusão no estudo foram: ter 60 anos ou mais e participar regularmente das atividades de um dos grupos há pelo menos dois meses. Não houve nenhuma recusa em participar do estudo.

Para traçar o perfil dessa população, foi aplicado um formulário com questões de múltipla escolha contendo as seguintes variáveis: sexo, idade, procedência, escolaridade, estado civil, ocupação, renda, habitação, tempo de participação no grupo, relação com os familiares, comunicação intrafamiliar, autonomia financeira, auxílio nas despesas, autoavaliação da saúde, energia para realizar as atividades diárias, história clínica, uso de medicamentos, acompanhamento profissional, sexualidade, prática de exercícios físicos, alimentação, fumo, uso de álcool e atividades de lazer. As questões foram adaptadas a partir do questionário do estudo Saúde, Bemestar e Envelhecimento (SABE), elaborado no município de São Paulo. ${ }^{4}$ A coleta de dados foi realizada por meio de entrevista no período de fevereiro a março de 2009. Estatísticas descritivas e medidas de tendência central e dispersão foram utilizadas para descrição das variáveis com utilização do programa SPSS, versão 11.0. O projeto foi submetido ao Comitê de Ética da Faculdade de Medicina de Juazeiro (FMJ), sob o protocolo $\mathrm{n}^{\mathrm{0}}$ 2009/0034/FRSN, respeitando-se os termos da Resolução no 196/96, do Conselho Nacional de Saúde (CNS). ${ }^{5}$

\section{RESULTADOS}

Dentre os idosos convidados a participar do estudo, foram elegíveis 52 idosos do grupo SESC e 13 idosos do Grupo da Melhor Idade. O quantitativo de 65 idosos envolvidos neste estudo corresponde a $72,2 \%$ do total de idosos que participam regularmente dos grupos de convivência.

A tabela 1 apresenta número e percentual de dados sociodemográficos dos idosos entrevistados. Observa-se que entre os 65 idosos entrevistados, o predomínio foi do sexo feminino, com idade média foi de 69,82 anos ( $\mathrm{DP} \pm 6,62$ ), com grande parte do grupo com pessoas viúvas $(\mathrm{n}=29)$ e morando em casa própria. Em relação à ocupação, 73,8\% $(n=48)$ eram aposentados e $21,5 \%(\mathrm{n}=14)$ realizavam atividades do lar, sem nenhuma remuneração. Ainda participaram do estudo, um comerciante, uma artesã e uma auxiliar de enfermagem. 
Tabela 01 - Dados sociodemográficos dos idosos entrevistados. Iguatu, CE, 2009.

\begin{tabular}{|c|c|c|}
\hline Dados Sociodemográficos & $\mathrm{n}$ & $\%$ \\
\hline \multicolumn{3}{|l|}{ Sexo } \\
\hline Feminino & 59 & 90,8 \\
\hline Masculino & 06 & 9,2 \\
\hline \multicolumn{3}{|l|}{ Idade } \\
\hline $60-64$ anos & 20 & 30,8 \\
\hline $65-69$ anos & 17 & 26,1 \\
\hline 70-74 anos & 14 & 21,6 \\
\hline$>75$ anos & 05 & 21,5 \\
\hline \multicolumn{3}{|l|}{ Estado Civil } \\
\hline Solteiro & 07 & 10,8 \\
\hline Casado & 23 & 25,4 \\
\hline Viúvo & 29 & 44,6 \\
\hline Divorciado & 06 & 9,2 \\
\hline \multicolumn{3}{|l|}{ Escolaridade* } \\
\hline Nenhuma & 10 & 15,6 \\
\hline 1 a 3 anos & 17 & 26,6 \\
\hline 4 a 7 anos & 26 & 40,6 \\
\hline 8 anos ou mais & 11 & 17,2 \\
\hline \multicolumn{3}{|l|}{ Renda Familiar } \\
\hline Até 1 SM & 12 & 18,4 \\
\hline 1 a 2 SM & 20 & 30,8 \\
\hline 2 a 3 SM & 26 & 40,0 \\
\hline$>3 \mathrm{SM}$ & 07 & 10,8 \\
\hline \multicolumn{3}{|l|}{ Habitação } \\
\hline Própria & 57 & 87,7 \\
\hline Alugada & 05 & 7,7 \\
\hline Cedida & 03 & 4,6 \\
\hline
\end{tabular}

*1 indivíduo não respondeu.

Quanto à renda familiar mensal, 40\% $(\mathrm{n}=26)$ dos entrevistados possuem renda mensal de dois a três salários mínimos, 30,8\%(n=20) afirmaram variar de um a dois salários mínimos, 18,5\% ( $\mathrm{n}=$ 12) relataram ter renda familiar inferior a um salário mínimo e apenas $10,8 \%(n=7)$ possuem rendimento superior a três salários.
Os dados relacionados ao contexto familiar e as redes sociais de apoio aos idosos mostraram que $33,8 \%(n=22)$ dos idosos entrevistados residiam com os filhos e $20 \%(n=13)$ moravam sozinhos, e $87,3 \%(n=55)$ dos entrevistados consideraram como bom ou ótimo o apoio dos familiares para realização das atividades de rotina. 
Tabela 02 - Contexto familiar e as redes sociais de apoio na percepção dos idosos. Iguatu, CE, 2009.

\begin{tabular}{lcc}
\hline \multicolumn{1}{c}{ Contexto Familiar } & $\mathrm{n}$ & $\%$ \\
\hline Com quem moram (n=65) & 13 & 20,0 \\
$\quad$ Sozinho & 11 & 16,9 \\
Com Companheiro & 22 & 33,8 \\
$\quad$ Com Filhos & 11 & 16,9 \\
$\quad$ Com companheiro e filhos & 08 & 12,4 \\
$\quad$ Outros & & \\
Relação com os Familiares (n=63) & 19 & 30,2 \\
$\quad$ Otima & 36 & 57,1 \\
Boa & 08 & 12,7 \\
$\quad$ Regular & & \\
Comunicação com os Familiares (n=63) & 19 & 30,2 \\
$\quad$ Otima & 36 & 57,1 \\
$\quad$ Boa & 08 & 12,7 \\
$\quad$ Regular & & \\
Apoio Familiar para realização de atividades (n=63) & 28 & 44,4 \\
$\quad$ Otimo & 28 & 44,4 \\
$\quad$ Bom & 07 & 11,2 \\
$\quad$ Regular & & \\
Apoio financeiro no orçamento familiar (n=65) & 33 & 50,8 \\
$\quad$ São os únicos responsáveis pelo sustento da casa & 24 & 36,9 \\
$\quad$ Dividem os gastos com os familiares & 08 & 12,3 \\
$\quad$ Não contribuem financeiramente & & \\
\hline
\end{tabular}

Quando perguntados sobre sua situação de saúde, de acordo com a autoavaliação, os idosos se consideram em estado regular $(53,8 \%)$, demonstrando disposição boa ou regular para realizar atividades. Quase $80 \%$ fazem uso de medicação contínua e têm acompanhamento profissional. Dos agravos diagnosticados entre os idosos entrevistados, os mais frequentes foram: hipertensão, diabetes, problemas cardiovasculares, osteoporose, artrose e depressão. Os medicamentos mais consumidos rotineiramente são os anti-hipertensivos.

Quanto ao desenvolvimento de atividades de lazer extragrupo, foi relatado que ir à igreja, viajar e participar de festas são as atividades mais prazerosas e rotineiras. 
Tabela 03 - Frequência absoluta e percentual das condições e autoavaliação de saúde dos idosos entrevistados. Iguatu-CE, 2009.

\begin{tabular}{lcc}
\multicolumn{1}{c}{ Avaliação de Saúde } & $\mathrm{n}$ & $\%$ \\
\hline Autoavaliação & 10 & \\
$\quad$ Otima & 15 & 15,4 \\
Boa & 35 & 23,1 \\
Regular & 05 & 53,8 \\
$\quad$ Péssima & & 7,7 \\
Disposição para realizar atividades & 01 & \\
$\quad$ Excelente & 14 & 1,5 \\
Otima & 25 & 21,5 \\
Boa & 21 & 38,6 \\
Regular & 03 & 32,3 \\
Péssima & 01 & 4,6 \\
Não respondeu & & 1,5 \\
Diagnóstico confirmado de doença & 56 & 86,2 \\
Sim & 09 & 13,8 \\
Não & & \\
Uso de medicação contínua & 51 & 78,5 \\
Sim & 14 & 21,5 \\
Não & & 80,0 \\
Acompanhamento Profissional & 52 & 20,0 \\
$\quad$ Sim & 13 & 84,6 \\
Não & & 15,4 \\
Outras atividades de Lazer & 55 & \\
Sim & 10 & \\
Não & & \\
\hline
\end{tabular}

Observando a tabela 4, verificou-se que $55,4 \%(\mathrm{n}=36)$ dos idosos entrevistados consideram boa a qualidade de sua alimentação, não referem tabagismo ou consumo de bebidas alcoólicas e em sua maioria fazem atividade física.

Tabela 04 - Variáveis relacionadas ao estilo de vida de idosos frequentadores de grupo de convivência. Iguatu-CE, 2009.

\begin{tabular}{lcc}
\hline \multicolumn{1}{c}{ Hábitos de Vida } & $\mathrm{n}$ & $\%$ \\
\hline Qualidade da Alimentação & & \\
$\quad$ Otima & 14 & 21,5 \\
Boa & 36 & 55,4 \\
Regular & 15 & 23,1 \\
Fumo & & \\
$\quad$ Sim & 05 & 7,7 \\
$\quad$ Não & 60 & 92,3 \\
Bebida Alcoólica & 06 & 9,2 \\
$\quad$ Sim & 59 & 90,8 \\
Não & & \\
Prática de Exercícios & 59 & 90,8 \\
$\quad$ Sim & 06 & 9,2 \\
$\quad$ Não & &
\end{tabular}




\section{DISCUSSÃO}

No que tange às características demográficas dos grupos de convivência da terceira idade no município estudado, observa-se uma predominância de pessoas do sexo feminino, viúvas e de baixa escolaridade. Esta elevada frequência de participação feminina pode estar refletindo a composição demográfica dos idosos com maior probabilidade de sobrevida pelas mulheres. ${ }^{6}$ Pode-se supor também que as mulheres têm maior atenção com a saúde e com o autocuidado e que os idosos do sexo masculino participam menos de ações coletivas por questões socioculturais.

A alta frequência de viúvas pode ser explicada pela elevação progressiva na expectativa de vida da população feminina em detrimento $d a$ masculina. Esse fator tanto é relevante que o Ministério da Saúde instituiu, no ano de 2008, a política de saúde do homem, visando, dentre outros fatores, a diminuir a mortalidade masculina em todas as faixas etárias, a fim de aumentar a expectativa de vida desse grupo, que é sete anos menor que em mulheres. ${ }^{7}$ Estudo realizado em Goiânia mostra dados semelhantes aos do presente estudo, onde $45,3 \%$ dos idosos eram casados, $44 \%$ eram viúvos, $9,3 \%$ eram desquitados/divorciados e apenas $1,3 \%$ era solteiro. $^{8}$

No que diz respeito à escolaridade, quase $70 \%$ dos idosos possui até quatro anos de estudo. A baixa escolaridade foi característica destacada em estudo realizado com idosos de Fortaleza ${ }^{9}$ e em Belo Horizonte com grupo semelhante. ${ }^{10}$ Em um estudo realizado com 149 idosos institucionalizados no Distrito Federal, 32,2\% dos entrevistados nunca haviam estudado e apenas $1,4 \%$ possuía nível superior. ${ }^{11}$

A baixa escolaridade da população idosa foi confirmada pelo censo realizado com idosos responsáveis pelos domicílios brasileiros, que revelou que 59,4\% destes tinham, no máximo, três anos de estudo, resultado este influenciado pela alta proporção de responsáveis de 75 anos ou mais de idade analfabetos ou analfabetos funcionais. Enquanto $53,3 \%$ dos idosos no grupo de 60 a 64 anos tinham até três anos de estudos, $67,4 \%$ daqueles com 75 anos ou mais de idade foram considerados analfabetos funcionais. Ainda assim, houve significativa melhora no período intercensitário, provavelmente resultado dos programas federais de alfabetização de adultos executados nas últimas duas décadas. ${ }^{6}$

Em se tratando de questões de relacionamento com familiares e criação de novas redes sociais, observaram-se neste estudo $20 \%$ de idosos morando sozinhos, o que se mostra uma elevada frequência, se relacionado a alguns estudos. ${ }^{4,8,9}$ Sobre o relacionamento com os familiares, $57,1 \%$ $(\mathrm{n}=36)$ avaliaram como bom e em relação à comunicação com os mesmos, $30,2 \%(n=19)$ avaliaram como ótima.

Em pesquisa realizada na cidade de FortalezaCE, apenas $5 \%$ dos idosos residiam sozinhos, enquanto que $84 \%$ dividiam o lar com filhos e netos. ${ }^{7}$ Numa pesquisa realizada em Goiânia, segundo a qual $42,7 \%$ dos idosos residiam com filhos e netos, $33 \%$ moravam com o companheiro e apenas 5,3\% moravam sozinhos. ${ }^{8}$

O bom relacionamento familiar mostra-se recorrente nos estudos realizados com idosos. Estudos realizados na região Centro-Oeste da cidade de São Paulo e em Belo Horizonte corroboram esses dados. ${ }^{10,13}$

É importante que, no contexto familiar, o idoso tenha oportunidade de se comunicar livremente, relatando suas necessidades, transmitindo sua experiência e participando efetivamente das decisões. Desta forma, esse idoso se sentirá útil e inserido no contexto familiar, o que acaba por melhorar sua autoestima e qualidade de vida. A comunicação é um importante instrumento de interação social, devendo ser estimulada pelas redes sociais de apoio, mediante atividades educativas que proporcionem espaços amplos para a socialização. ${ }^{14}$

Dentro dessas redes sociais, os grupos de terceira idade são espaços importantes que permitem a interação social, devendo criar 
estratégias eficazes de estímulo a comunicação e valorização da autoestima do idoso. Contudo, é em casa que o idoso permanece a maior parte do tempo, tornando necessário haver uma comunicação ampla e respeitosa, permitindo que o idoso permaneça inserido na dinâmica familiar e possa verbalizar suas necessidades e opiniões.

Sobre a questão econômica, os idosos entrevistados afirmaram que tinham dinheiro suficiente para o suprimento de suas necessidades básicas. Esse dado difere do encontrado de estudos realizados no município de São Paulo e Belo Horizonte, onde idosos entrevistados não possuem renda suficiente para suprir suas necessidades. ${ }^{10,15}$

As condições financeiras dos idosos interferem nas suas preocupações e desejos. Esta divergência de opinião a respeito da quantidade de dinheiro suficiente pode ser explicada pelo fato de que, para alguns deles, as metas existenciais se referem a simplesmente sobreviver, enquanto que para outros, refletem a busca da concretização de sonhos de diferentes dimensões. ${ }^{16}$

Nas últimas décadas tem-se observado que muitos idosos têm sido responsáveis por parcelas significativas do orçamento familiar. É recorrente observar idosos que acabam por assumir a responsabilidade financeira do lar, passando muitas vezes a prover o sustento de filhos e netos. No contexto nacional, os idosos acabam por ter perdas salariais significativas no momento de sua aposentadoria, em contraponto aos inúmeros gastos comuns nessa fase da vida, especialmente com medicamentos, exames e consultas.

Em relação ao auxílio no orçamento familiar observou-se que $50,8 \%(\mathrm{n}=33)$ sustentavam sozinhos suas residências. Segundo a Síntese dos Indicadores Sociais do IBGE, em 2008, os idosos contribuem de maneira decisiva para o orçamento da maior parte das famílias brasileiras. Em 53\% dos domicílios do país, a contribuição dos idosos com 60 anos ou mais representa mais da metade do total da renda domiciliar. Na Região Nordeste, em $63,5 \%$ dos domicílios os idosos representam mais da metade da renda. Na zona rural nordestina este percentual de contribuição no orçamento familiar é ainda mais significativo. Em $73 \%$ dos domicílios da zona rural, os idosos tinham participação superior a $50 \%$ da renda familiar. ${ }^{17}$

A aposentadoria possibilitou ao idoso a garantia de um rendimento mínimo para o atendimento de suas necessidades básicas. Embora mais vulnerável às despesas com remédios e demais tratamentos de saúde, esta parcela da população possui hoje melhores condições financeiras do que os mais jovens. Isto acaba por gerar uma redistribuição intergeracional da renda do idoso. ${ }^{18}$

Sobre a autopercepção de saúde, consideram que têm boa saúde e disposição regular para realizar atividades. Esses resultados se aproximam a de uma pesquisa que avaliou os determinantes da autopercepção de saúde entre idosos do município de São Paulo. Nele, 48,9\% dos idosos consideraram sua saúde como regular 25,7\% consideraram ter boa saúde, $6,6 \%$ avaliaram como ótima sua condição de saúde e 17,8\% consideraram como má sua condição de saúde. ${ }^{19}$

A autopercepção de saúde contempla aspectos físicos, cognitivos e emocionais. Trata-se de um poderoso indicador de mortalidade. Segundo Rodrigues \& Alves (2005), ${ }^{19}$ as pessoas que consideram ruim seu estado de saúde apresentam maior risco de mortalidade por todas as causas, em comparação com aquelas que relatam ter uma excelente saúde.

A disposição para a realização das atividades rotineiras está diretamente relacionada com a condição de saúde dos idosos. ${ }^{16} \mathrm{O}$ envelhecimento passa a ser vivenciado concretamente com o declínio de força física e a falta de disposição para realizar atividades comuns de outrora, tanto no âmbito profissional como nos cuidados com a casa.

O consumo de medicamentos de uso contínuo nesse grupo etário é alto, decorrente das doenças crônicas já diagnosticadas Em pesquisa realizada na cidade de São Paulo, 94\% dos idosos tinham acompanhamento profissional de rotina. ${ }^{20}$ Estudo realizado nesta mesma metrópole sobre o 
consumo de medicamentos revelou que $88,6 \%$ $(\mathrm{n}=320)$ utilizavam rotineiramente fármacos. ${ }^{21}$

Em um estudo realizado na cidade de Curitiba, 47,2\% dos idosos utilizavam antihipertensivos e medicamentos de ação cardiovascular, 47,1\% utilizavam rotineiramente analgésicos e anti-inflamatórios, 37,7\% faziam uso de polivitamínicos, $28,3 \%$ tomavam medicamentos de reposição hormonal e $26,4 \%$ faziam uso de protetores gástricos. ${ }^{22}$

Dessa forma, verifica-se que o consumo de medicamentos entre os idosos é muito alto, tendência que acompanha o processo de envelhecimento com o surgimento de doenças crônicas não-transmissíveis e suas comorbidades. ${ }^{21}$

O acompanhamento da saúde do idoso é de suma importância, devendo ser realizado rotineiramente por um profissional capacitado. Isto permite um diagnóstico precoce de complicações, estímulo ao autocuidado e orientações sobre práticas saudáveis especificas para este grupo etário.

De acordo com Santos $\&$ Barros, ${ }^{13}$ as atividades de lazer são consideradas como práticas saudáveis pela Organização Mundial de Saúde . Observa-se que os entrevistados participam efetivamente de ações de lazer. A participação dos idosos nestas ações estimula a inclusão social, cria novas redes sociais e melhora a autoestima e a qualidade de vida nesta fase da vida.

A alimentação pode ser considerada um importante marcador de saúde do idoso. Diversos fatores podem influenciar na qualidade $d a$ alimentação nesse grupo etário. Além das alterações decorrentes do envelhecimento, é frequente o uso de múltiplos medicamentos que influenciam na ingestão de alimentos, digestão, absorção e utilização de diversos nutrientes, o que pode comprometer o estado de saúde e a necessidade nutricional do indivíduo idoso. ${ }^{23}$

Poucos idosos relataram tabagismo ou consumo de bebida alcoólica. Em um estudo sobre os fatores de risco para doença arterial em idosos, realizado no município de São Paulo, observou-se que entre os 100 idosos envolvidos, $18 \%$ eram fumantes..$^{20}$

A elevada frequência de prática esportiva entre os idosos entrevistados deve-se aos grupos de convivência em que estes se encontram inseridos, que oferecem prática regular de atividade física. O município ainda dispõe de academias na comunidade organizada pelo corpo de bombeiros, que é um serviço gratuito e aberto ao público de todas as faixas etárias.

\section{CONCLUSÕES}

Ao avaliar os grupos de convivência de idosos em Iguatu-CE, foi possível constatar uma predominância do sexo feminino, elevada frequência de viúvos e aposentados. A renda familiar pode ser considerada acima da média da população idosa.

Observou-se também que os idosos que participam dos grupos de convivência afirmaram ter uma relação satisfatória com seus familiares. A respeito da manutenção do lar, boa parte destes colabora significativamente nas despesas da casa, chegando a arcar completamente com os custos do grupo familiar.

No que diz respeito à autoavaliação de saúde, grande parte dos idosos entrevistados considera ter um envelhecimento saudável, com satisfatórios níveis de saúde e disponibilidade para realizar seu autocuidado e outras atividades diárias.

O processo de participação nos grupos significa, para os idosos, uma forma de voltar para a sociedade, pois na maioria das vezes, quando se deparam com a velhice, enfrentam graves problemas, devido à perda de alguém próximo, como amigos ou parentes, incluindo-se também a troca do trabalho pela aposentadoria, do reconhecimento social, entre outros. A inclusão dos idosos nos grupos de convivência lhes possibilita inúmeras descobertas, no entanto oferece a essas pessoas a oportunidade de saírem de casa e se integrarem a projetos coletivos, que reforçam as redes sociais de apoio, fortalecem a autonomia e abrem espaço para novos projetos de vida. 
Por ser uma experiência salutar, cabe aos gestores e à sociedade efetivar as políticas públicas de promoção à saúde do idoso, envolvendo diversos setores da sociedade, em busca de oferecer uma rede de serviços que promovam a qualidade de vida e o bem-estar a este grupo etário.

Torna-se importante envolver um maior número de idosos nesses grupos, capacitar seus

\section{REFERÊNCIAS}

1. Santos JS, Barros M D. de A. Idosos do Município do Recife, Estado de Pernambuco, Brasil: uma análise da morbimortalidade hospitalar. Epidemiol Serv Saúde 2008; 17(3): 177186.

2. Pereira RS, Curioni CC, Veras R. Perfil demográfico da população idosa no Brasil e no Rio de Janeiro em 2002. Textos sobre Envelhecimento 2003; 6(1): 43-59.

3. Instituto Brasileiro de Geografia e Estatística. Contagem da população 2007. Disponível em: http://www.ibge.gov.br/cidadesat/ topwindow.htm?1. Acesso em : 20 de mar, 2009.

4. Lebrão ML, Duarte, YA de O. SABE: saúde, Bem-estar e envelhecimento no município de São Paulo: uma abordagem inicial. Brasília: OPAS/MS; 2003.

5. Brasil. Ministério da Saúde. Conselho Nacional de Saúde. Normas de pesquisa envolvendo seres humanos. Res CNS 196/96. Bioética 1996; 4 Suppl: 15-25.

6. . Ministério do Planejamento, Orçamento e Gestão. Instituto Brasileiro de Geografia e Estatística - IBGE. Perfil dos idosos responsáveis pelo domićlío no Brasil. Rio de Janeiro: IBGE; 2002.

$7 . \quad$. Ministério da Saúde. Secretaria de atenção à saúde. Departamento de ações programáticas estratégicas. Política Nacional de atenção Integral à Saúde do Homem. Brasília; 2008.

8. Araújo LAO, Bachion MM. Programa saúde da Família: perfil de idosos assistidos por uma equipe. Rev Brás Enferm 2004; 57(5): 586-90.

9. Menezes TN, Lopes FJM, Marucci MFN. Estudo domiciliar da população idosa de Fortaleza/CE: aspectos metodológicos e características sócio-demográficas. Rev. Bras. Epidemiol 2007; 10(2): 168-77. responsáveis e oferecer condições adequadas para o desenvolvimento dessas práticas de promoção à saúde. A efetivação de parcerias com a Estratégia Saúde da Família, com o Corpo de Bombeiros, com instituições governamentais e nãogovernamentais podem propiciar uma maior oferta de atividades que sirvam como atrativo para captação de mais componentes para estes grupos.

10. Borges PLC; Bretas RP;Azevedo SF de; Barbosa JMM. Perfil dos idosos freqüentadores de grupos de convivência em Belo Horizonte, Minas Gerais, Brasil. Cad Saude Publica 2008; 24(12): 2798-2808.

11. Danilow MZ, et al et al. Perfil epidemiológico, sociodemográfico e psicossocial de idosos institucionalizados do Distrito Federal. Comunicação em Ciências da Saúde 2007; 18(1): 9 -16.

12. Ministério da Saúde( Brasil). Pacto pela Saúde: Colônia Leopondina; Ministério da Saúde, 2007. [Acesso em: 15 de out. 2008] Disponível em: http://portal.saude.al.gov.br/suvisa/files/ Pacto\%20pela\%20Sa\%C3\%BAde\%202007\%20$\% 20$ Coqueiro\%20Seco.pdf

13. Santos J S, Barros MD A. Idosos do Município do Recife, Estado de Pernambuco, Brasil: uma análise da morbimortalidade hospitalar. Epidemiol Serv Saúde 2008;17(3):177-186.

14. Guidetti AA. Pereira AS. A importância da educação na socialização dos idosos. Revista de Educação2008: 119-136.

15. Louvison MCP, Lebrão ML, Duarte YAO, Laurenti R. Desigualdades nas condições de saúde e no uso de serviços entre as pessoas idosas no município de São Paulo: uma análise de gênero e renda. Saúde Coletiva 2008; 5(24):189-194.

16. Silva EV, Martins F, Bachion MM, Nakatani AYK. Percepção de idosos de um centro de convivência sobre envelhecimento. Reme 2006; 10(1): 46-53.

17. IBGE. Instituto Brasileiro de Geografia e Estatística. Censo e Sociedade: Estatísticas para a Cidadania. Revistas do Censo 2000 [ acesso em 08 Out 2008] . Disponível em: < http:// www.ibge.gov.br/censo/>

18. Coutrim RME. Idosos trabalhadores: perdas e ganhos nas relações intergeracionais. Sociedade e Estado.2006; 21(2):367-390. 
19. Rodrigues RN, Alves LC. Determinantes de autopercepção de saúde entre idosos do município de São Paulo. Rev Panam Salud Publica 2005; 17(5-6): 333-341.

20. Silva AL, Sales PC. Participação familiar na prevenção primária dos fatores de risco para doença arterial coronária em idosos. Saúde Coletiva 2008; 5(22): 110-115.
21. Menezes FG, Wadt NSY, Sousa R, Santos AS. Consumo medicamentoso em idosos na região centro-oeste da cidade de São Paulo. Saúde Coletiva 2008; 5(22): 126-130.

22. Penteado PTP. O uso de medicamentos por idosos. Visão Acadêmica. 2002; 3(1): 35-42.

23. Campos MTFS, Monteiro JBR.; Ornelas APRC. Fatores que afetam o consumo alimentar e a nutrição do idoso. Rev. Nutr 2000; 13(3):157-165.

Recebido: 30/11/2009

Revisado: 06/6/2010

Aprovado: 28/8/2010 
